\title{
Absence of bilinear condensate in three-dimensional QED
}

\section{Nikhil Karthik*}

Florida International University

E-mail: nkarthik@fiu.edu

\section{Rajamani Narayanan}

Florida International University

E-mail: rajamani.narayananefiu.edu

There are plausibility arguments that QED in three dimensions has a critical number of flavors of massless two-component fermions, below which scale invariance is broken by the presence of bilinear condensate. We present numerical evidences from our lattice simulations using dynamical overlap as well as Wilson-Dirac fermions for the absence of bilinear condensate for any even number of flavors of two-component fermions. Instead, we find evidences for the scale-invariant nature of three-dimensional QED.

34th annual International Symposium on Lattice Field Theory

24-30 July 2016

University of Southampton, UK

\footnotetext{
* Speaker.
} 


\section{Introduction}

Parity-invariant $\mathrm{QED}_{3}$ with $2 N_{f}$ flavors of massless two-component fermions coupled to threedimensional non-compact Abelian gauge-fields has been studied in the past as a quantum field theory which can be tuned to be conformal or to have a mass-gap by changing $N_{f}$. The question is the following - is there a critical number of flavors of two-component fermions $2 N_{f}$ below which massless $\mathrm{QED}_{3}$ in a finite box of length $\ell$ generates other low-energy length scales which are independent of $\ell$ as $\ell \rightarrow \infty$ ? One such low-energy length scale that is of interest is the bilinear condensate $\Sigma$ which, if non-zero, governs the following scaling of the low-lying eigenvalues $\lambda_{i}$ of the massless Dirac operator:

$$
\lambda_{i}=\frac{z_{i}}{\Sigma} \frac{1}{\ell^{3}}
$$

where $z_{i}$ are universal numbers depending only on the symmetries of the Dirac operator, and can be obtained from a random matrix model with the same symmetries (refer [1] for such a model corresponding to $\mathrm{QED}_{3}$ ). In this talk, based on our publications [2, 3], we primarily address the existence of $\Sigma$ for small $N_{f}(=1,2,3,4)$ by asking if $\lambda \sim \ell^{-(1+p)}$ with $p=2$. We summarize the status of the understanding of the critical $N_{f}$ before our studies in Figure 1 (see [2] and references therein, for a complete literature survey). The analytical computations, each with their own limitations, suggested that the critical $N_{f}$ lie between 0 and 4 . The previous lattice studies suggested that it could be 1 or 2 .

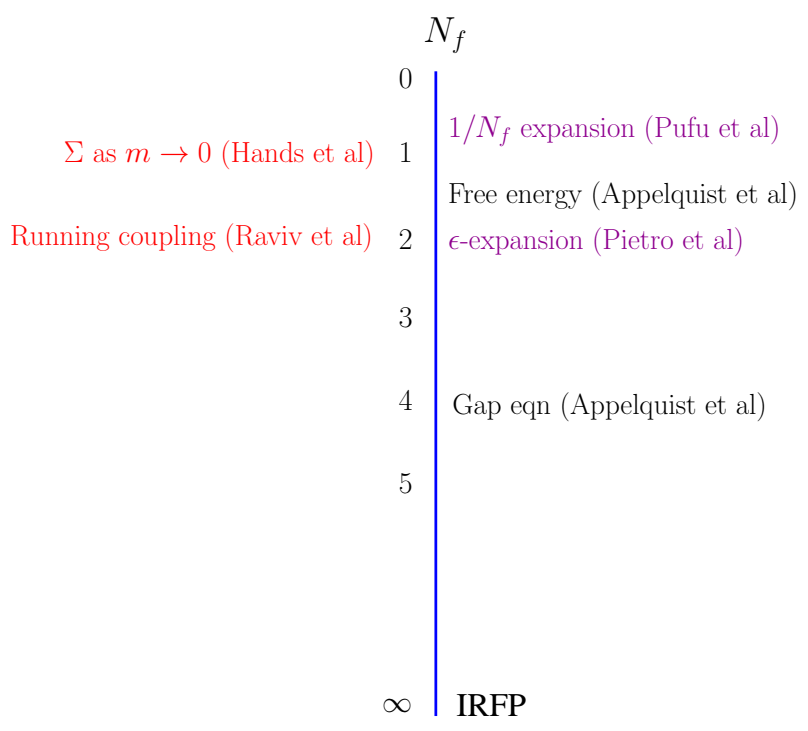

Figure 1: Few representative older calculations [4, 5, 6, 7, 8, 9] of the critical value of $N_{f}$ below which bilinear condensate exists. The large- $N_{f}$ computation points to an infra-red fixed point. Various perturbative calculations as well as approximate solutions to the gap equation have been carried out to investigate the stability of the infra-red fixed point. These calculations suggest the critical value might lie anywhere between 0 and 4. The previous non-perturbative lattice studies of $\mathrm{QED}_{3}$ suggest this critical value might be 1 or 2.

\section{Lattice details}

We regulated $\mathrm{QED}_{3}$ in a finite box of physical volume $\ell^{3}$ using $L^{3}$ lattices. The lattice coupling 
appearing in the gauge action is $\beta=L / \ell$; the continuum limit at a fixed physical length $\ell$ is taken by extrapolating to $L \rightarrow \infty$. We regulated the two flavors of massless two-component fermions in a parity-invariant way using the Wilson-Dirac as well as overlap fermions. The fermion propagator $G$ for the parity-preserving Wilson-Dirac fermion is

$$
G^{-1}=\left[\begin{array}{cc}
0 & X \\
-X^{\dagger} & 0
\end{array}\right] \quad ; \quad X=C_{n}+B-m_{t} .
$$

$C_{n}$ is the two-component naive Dirac operator, $B$ is the Wilson term and $m_{t}$ is tuned such that the lowest eigenvalue $\lambda_{1}$ of $i G^{-1}$ is minimum. We further improved it by adding a SheikholeslamiWohlert term and by using HYP smeared links in the Dirac operator. The fermion propagator $G$ for the overlap fermion, which has the full $\mathrm{U}\left(2 N_{f}\right)$ symmetry even at finite lattice spacing, is given in terms of a unitary matrix $V=\left(X^{\dagger} X\right)^{-1} X$ as ${ }^{1}$

$$
G^{-1}=\left[\begin{array}{cc}
0 & \frac{1-V}{1+V} \\
\frac{1-V}{1+V} & 0
\end{array}\right] .
$$

We define the "eigenvalues of the Dirac operator" in either case to be the eigenvalues $\lambda_{i}$ of $i G^{-1}$ which are real. We used standard HMC for generating $\sim 500-1000$ independent gauge configurations at all the simulation points $(4 \leq \ell \leq 250)$. Using Wilson-Dirac fermions we studied $N_{f}=1,2,3$ and 4 . With the overlap fermion, we studied $N_{f}=1$. At each $\ell$, we used multiple $L^{3}$ lattices $(12 \leq L \leq 24)$ in order to take the continuum limits.

\section{Evidence from $\ell$-scaling of the low-lying eigenvalues of Dirac operator}
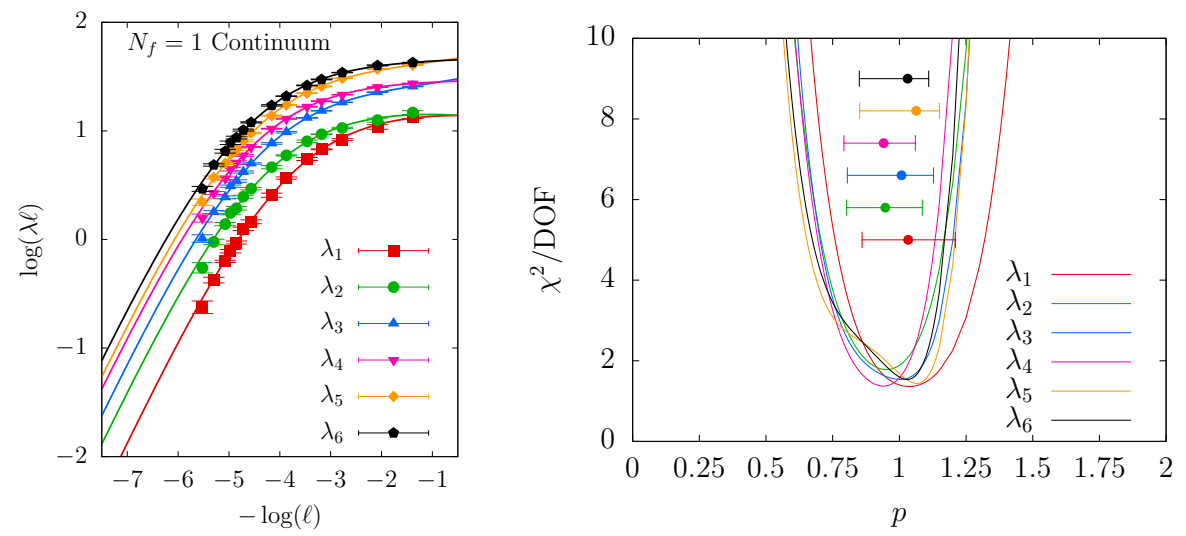

Figure 2: On the left panel, the $\ell$ dependence of the six low-lying, continuum extrapolated, eigenvalues of the overlap operator is shown. The Padé approximations to their $\ell$ dependence with $p=1$ are shown as the solid curves. On the right panel, the likelihood of different values of the exponent $p$, measured using the $\chi^{2} /$ DOF for the best fit of the Padé approximation with various values of $p$ to the finite $\ell$ data, is shown.

\footnotetext{
${ }^{1}$ The Wilson mass $m_{t}=1$ in overlap simulations
} 
In a finite physical box, the spectrum of the Dirac operator is discrete. Thus, one can talk about the $\ell$-dependence of the individual low-lying eigenvalues. As we noted in the introduction, the $i$-th low-lying eigenvalue $\lambda_{i}$ will scale as $\ell^{-3}$ when there is a condensate $\Sigma$. If $\ell^{-3}$ scaling is not found, we can conclude that a bilinear condensate is absent and instead we can obtain the mass anomalous dimension of the scale-invariant theory; since $\lambda$ has an engineering dimension of mass, the mass anomalous dimension $\gamma_{m}$ is $p$ if $\lambda \sim \ell^{-p-1}$ and $p<1$.

In the left panel of Figure 2, we show the dependence of the continuum extrapolated values of $\lambda_{i} \ell$ as a function of $1 / \ell$ for the six low-lying eigenvalues of the overlap operator in a log-log plot. At any finite $\ell$ that we studied, the slope $\frac{d \log (\lambda \ell)}{d \log (1 / \ell)}$ is less than 2 , the value that is expected if $\Sigma \neq 0$. In fact, it is less than 1 . We estimate the exponent of the power-law that would be seen as $\ell \rightarrow \infty$ by describing the $\ell$-dependence of our data by

$$
\lambda \ell=\ell^{-p} F(1 / \ell)
$$

with an unknown scaling correction $F$. We approximate $F$ by a [1/1] Padé approximant. We find it numerically stable to write the Padé approximant in terms of $\tanh (1 / \ell)$. The best fits of the above ansatz with $p=1$ to the data are shown by the solid curves in the left panel of Figure 2. In the right panel, we show the $\chi^{2} / \mathrm{DOF}$ for such fits to the six low-lying eigenvalues as a function of the exponent $p$. The value $p=2$ is clearly ruled out, which implies the absence of a condensate. Assuming the theory does not generate other length scales as well, we can estimate the mass anomalous dimension $\gamma_{m}=p$ of the theory to be 1.0(2) from the same plot. Further, we support the correctness of our result by comparing the $\ell$-dependence of the continuum extrapolated low-lying eigenvalues of the two different lattice Dirac operators in Figure 3. A perfect agreement between the Wilson-Dirac and the overlap formalisms is seen. Due to such an agreement, we study the $N_{f}=2,3,4$ cases using only the Wilson-Dirac fermion.

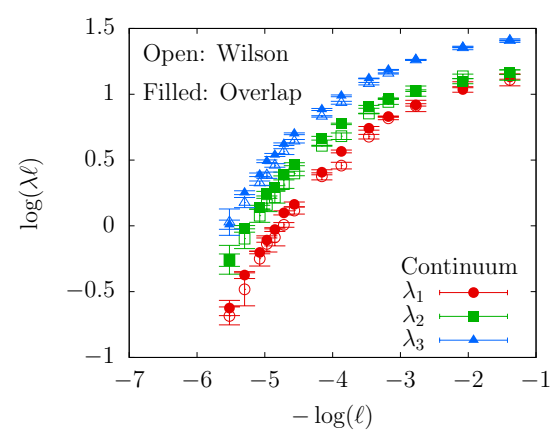

Figure 3: The plot compares the $\ell$-dependence of the first three low-lying eigenvalues, after taking the continuum limit, using Wilson fermions (open symbols) and overlap fermions (filled symbols) for the $N_{f}=1$ case.

In Figure 4, we show the $\ell$-dependence of the continuum extrapolated smallest eigenvalue for different number of flavors $N_{f}=1,2,3$ and 4 . The eigenvalues scale with a smaller exponent $p$ as $N_{f}$ increases, consistent with the expectation that if $N_{f}=1$ does not have a bilinear condensate, the $N_{f}=2,3,4$ also would not. Thus $\mathrm{QED}_{3}$ does not have a bilinear condensate for all non-zero $N_{f}$. Again, assuming this means that $\mathrm{QED}_{3}$ is scale-invariant for all $N_{f}$, we estimate the mass 


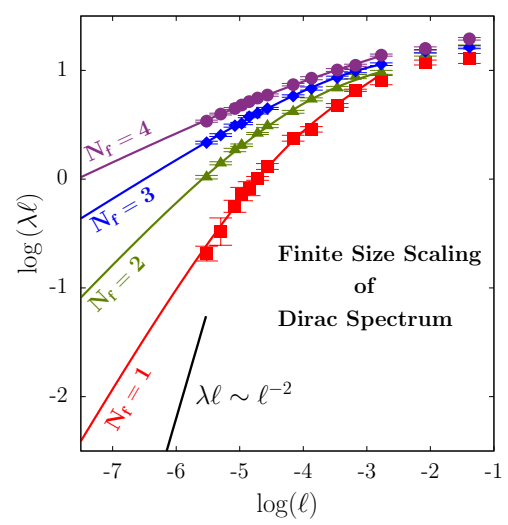

Figure 4: The $\ell$-dependence of the smallest eigenvalue of the Wilson-Dirac operator for $N_{f}=1,2,3$ and 4 . The expected scaling when a bilinear condensate is present, $\lambda \ell \sim \ell^{-2}$, is shown by the black straight line in this $\log$-log plot. The exponent $p$ for the asymptotic $\ell$-scaling seems to decrease as $1 / N_{f}$.

anomalous dimension to be $\gamma_{m}=1.0(2), 0.6(2), 0.37(6)$ and $0.28(6)$ for $N_{f}=1,2,3,4$ respectively. Surprisingly, this agrees with an analytical calculation [10] of $\gamma_{m}$ to $\mathscr{O}\left(1 / N_{f}^{2}\right)$ where no assumption about bilinear condensate is made; the analytical values are $\gamma_{m}=1.19,0.56,0.37$ and 0.28 for $N_{f}=1,2,3,4$ respectively.
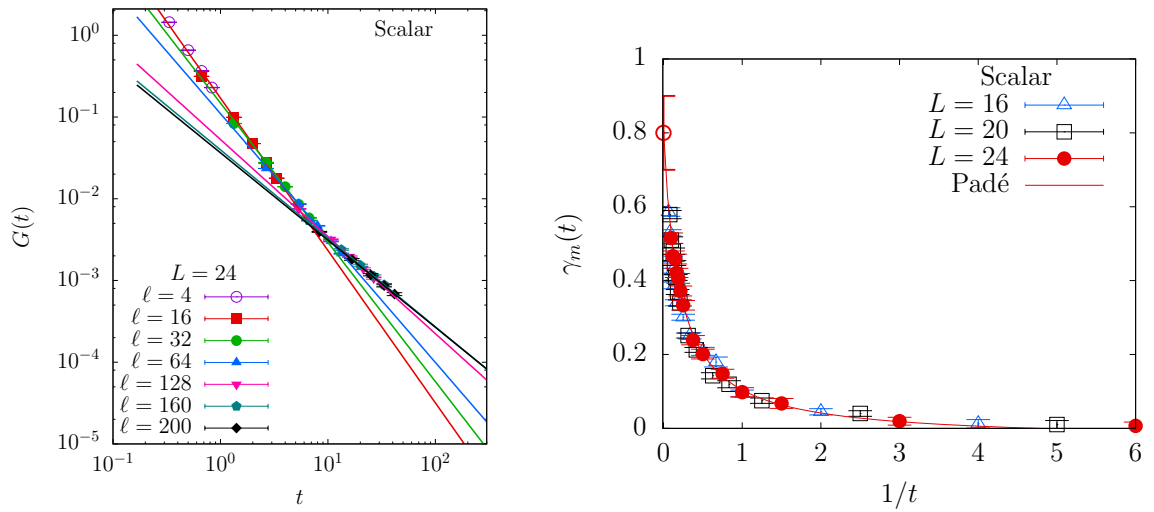

Figure 5: (Left) The zero spatial momentum scalar correlator $G(t)=\langle\Sigma(0) \Sigma(t)\rangle$ as a function of temporal separation $t$. The different lines are tangents to the correlator, with slope $k(t)$, at various $t$ on the log-log plot. (Right) The mass anomalous dimension given by $\gamma_{m}(t)=1-k(t) / 2$ is plotted as a function of the scale $t$.

The other way to obtain the mass anomalous dimension is to study the scalar correlator $G(t)=$ $\langle\Sigma(0) \Sigma(t)\rangle$ projected to zero spatial momentum. The correlator is shown as a function of the temporal separation $t$ in the left panel of Figure 5. The first thing to notice is the concave-up nature of the correlator. This indicates the absence of a mass-gap, thereby ruling out the presence of another length scale in addition to a bilinear condensate. The slope on the log-log plot, $k(t)=$ $\frac{d \log (G(t))}{d \log (t)}$, is related to a scale dependent mass anomalous dimension $\gamma_{m}(t)$ as $\gamma_{m}(t)=1-k(t) / 2$. This is shown as a function of $1 / t$ in the right panel of Figure 5 . The mass anomalous dimension 
at the IR fixed point to which $\mathrm{QED}_{3}$ with $N_{f}=1$ flows to, is $\gamma^{*}=\lim _{t \rightarrow \infty} \gamma_{m}(t)$. We estimate by an extrapolation that $\gamma^{*}=0.8(1)$. This is consistent with the estimate 1.0(2) from the eigenvalues described above. The agreement between two different approaches to $\gamma^{*}$ serves as a cross-check.

\section{Evidence from Inverse Participation Ratio and number variance}
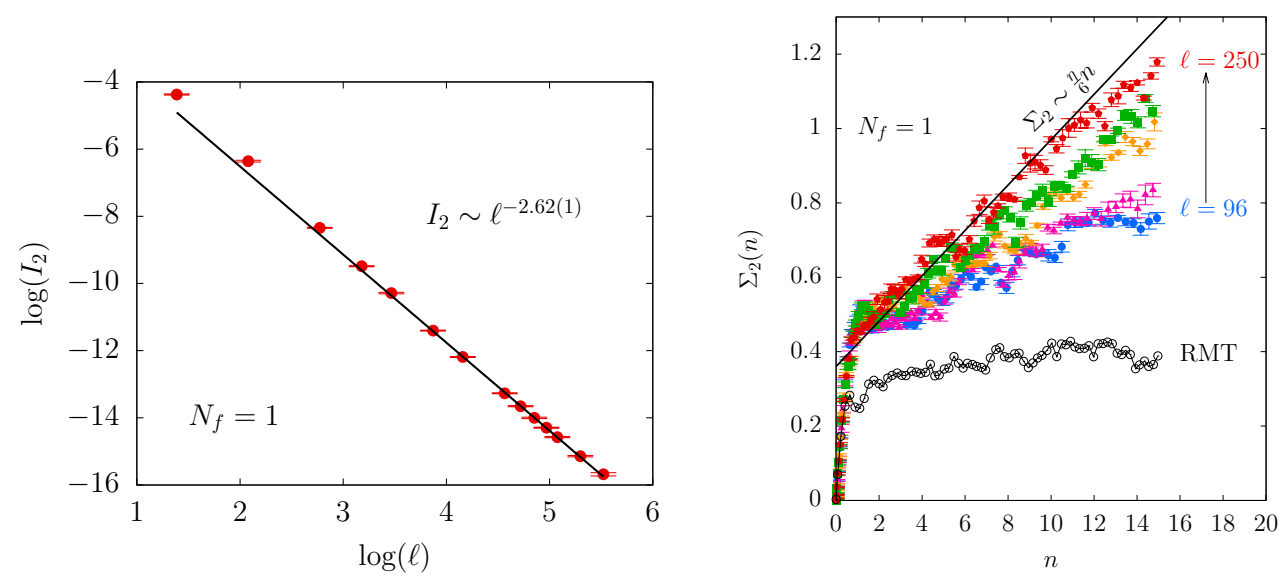

Figure 6: (Left) The $\ell$-scaling of the inverse participation ratio $I_{2}$ for $N_{f}=1$. The critical exponent of the scaling is $\eta=0.38(1)$. (Right) The number variance $\Sigma_{2}$ is shown as a function of $n$. A disagreement with nonchiral random matrix model (black points) is seen. Instead, a critical linear rise is seen, whose slope approaches $\eta / 6$ shown as the black solid line.

The Inverse Participation Ratio (IPR) is defined as

$$
I_{2} \equiv\left\langle\int\left(\psi_{\lambda}^{*}(x) \psi_{\lambda}(x)\right)^{2} d^{3} x\right\rangle
$$

where $\psi_{\lambda}$ is the normalized eigenvector corresponding to the eigenvalue $\lambda$. In random matrix models, which are ergodic, $I_{2} \sim \ell^{-3}$. Thus, if the theory has a condensate, the low-lying eigensystem of the Dirac operator would be described by a random matrix model. Thus the IPR corresponding to the low-lying eigenvalues should show a $\ell^{-3}$ scaling. This is another test for the presence of $\Sigma$. Instead, if the theory is scale-invariant, the finite size scaling of IPR would be $I_{2} \sim \ell^{-3+\eta}$, where $\eta$ is a critical exponent. The exponent $\eta$ is related to a quantity called number variance $\Sigma_{2}$ which measures correlations between the eigenvalues. The number variance $\Sigma_{2}(n)$ is defined as the variance of the number of eigenvalues below a value $\lambda$ which on the average contains $n$ eigenvalues. In ergodic random matrix models, $\Sigma_{2}(n) \sim \log (n)$. For a critical theory, $\Sigma_{2}(n) \sim(\eta / 6) n$, where $\eta$ is the critical exponent from the IPR [11].

In the left panel of Figure 6, we have shown the $\ell$-scaling of IPR for $N_{f}=1$. For large $\ell$, the onset of scaling is clearly seen. The scaling is $I_{2} \sim \ell^{-2.62(1)}$. Firstly, this rules out the ergodic $\ell^{-3}$ scaling. The theory has a non-zero critical exponent $\eta=0.38(1)$. As explained above, in a critical theory, $\eta$ should satisfy a critical relation to the slope of number variance. In the right panel of Figure 6, we have shown $\Sigma_{2}(n)$ as a function of $n$. Again, clearly there is a disagreement with the 
expectation from the nonchiral random matrix theory thereby ruling out condensate in another way. We see a linear rise in $\Sigma_{2}(n)$ indicating a critical behavior. As $\ell$ is increased, the slope of the linear rise seems to approach $\eta / 6$, as shown by the black line in the figure. Thus, both the IPR and $\Sigma_{2}$ show critical behavior, and also they satisfy the critical relation between the two.

\section{Conclusions}

In this talk, we presented convincing numerical evidences for the absence of a bilinear condensate for all $N_{f}$. Instead, we found evidences for $\mathrm{QED}_{3}$ to be scale-invariant, and we estimated the mass anomalous dimension at the infra-red fixed point at various $N_{f}$. In another work [12], we established the presence of a condensate in the 't Hooft limit using the same methods we described here. This suggests an interesting phase diagram in the $\left(N_{f}, N_{c}\right)$ plane whose one side is conformal while the other side has a mass-gap, providing a powerful system to understand the generation of mass in QFTs. We aim to present results on this in a future Lattice meeting.

The authors acknowledge partial support by the NSF under grant number PHY-1205396 and PHY-1515446.

\section{References}

[1] J. J. M. Verbaarschot and I. Zahed, Random matrix theory and QCD in three-dimensions, Phys. Rev. Lett. 73 (1994) 2288-2291, [hep-th/9405005].

[2] N. Karthik and R. Narayanan, Scale-invariance of parity-invariant three-dimensional QED, Phys. Rev. D94 (2016) 065026, [1606.04109].

[3] N. Karthik and R. Narayanan, No evidence for bilinear condensate in parity-invariant three-dimensional QED with massless fermions, Phys. Rev. D93 (2016) 045020, [1512 . 02993].

[4] T. W. Appelquist, M. J. Bowick, D. Karabali and L. C. R. Wijewardhana, Spontaneous Chiral Symmetry Breaking in Three-Dimensional QED, Phys. Rev. D33 (1986) 3704.

[5] T. Appelquist, A. G. Cohen and M. Schmaltz, A New constraint on strongly coupled gauge theories, Phys. Rev. D60 (1999) 045003, [hep-th/9901109].

[6] L. Di Pietro, Z. Komargodski, I. Shamir and E. Stamou, Quantum Electrodynamics in $d=3$ from the It $\hat{t}_{t}$ Expansion, Phys. Rev. Lett. 116 (2016) 131601, [1508.06278].

[7] S. M. Chester and S. S. Pufu, Anomalous dimensions of scalar operators in $Q E D_{3}, J H E P(08$ (2016) 069, [1603.05582].

[8] S. J. Hands, J. B. Kogut, L. Scorzato and C. G. Strouthos, Non-compact QED(3) with $N(f)=1$ and $N(f)=4$, Phys. Rev. B70 (2004) 104501, [hep-lat/ 0404013$].$

[9] O. Raviv, Y. Shamir and B. Svetitsky, Nonperturbative beta function in three-dimensional electrodynamics, Phys. Rev. D90 (2014) 014512, [1405.6916].

[10] J. A. Gracey, Electron mass anomalous dimension at $O(1 /(N f(2))$ in quantum electrodynamics, Phys. Lett. B317 (1993) 415-420, [hep-th/9309092].

[11] V. Chalker, J.T. amd Kravtsov and I. Lerner, Spectral rigidity and eigenfunction correlations at the Anderson transition, Pis'ma v ZhETF 64 (1996) 355-360.

[12] N. Karthik and R. Narayanan, Bilinear condensate in three-dimensional large- $N_{c} Q C D$, Phys. Rev. D94 (2016) 045020, [1607.03905]. 